\section{Case Reports in Ophthalmology}

Case Rep Ophthalmol 2020;11:606-611

DOI: 10.1159/000509629

Published online: November 10, 2020 (c) 2020 The Author(s)

Published by S. Karger AG, Basel www.karger.com/cop

\title{
Acute Bilateral Uveitis, Hypotony, and Cataracts Associated with Ipilimumab and Nivolumab Therapy: Optical Coherence Tomography Angiography Findings
}

\author{
Jessica C. Lee ${ }^{a} \quad$ Ghadeer Al-Humimat $^{\mathrm{a}-\mathrm{c}}$ Karanjit S. Kooner ${ }^{\mathrm{a}, \mathrm{c}}$ \\ aDepartment of Ophthalmology, University of Texas Southwestern Medical Center, \\ Dallas, TX, USA; 'bepartment of Ophthalmology, King Hussein Medical Center, \\ Amman, Jordan; 'Department of Ophthalmology, Veterans Affairs North Texas Health \\ Care System, Dallas, TX, USA
}

\section{Keywords}

Ipilimumab · Nivolumab · Uveitis · Hypotony · Cataracts · Optical coherence tomography angiography

\begin{abstract}
Immune checkpoint inhibitors are increasingly being used for the treatment of several malignancies. In rare cases, patients develop disabling ophthalmic side effects such as dry eyes, episcleritis, keratitis, uveitis, inflammatory orbitopathy, myasthenia gravis, macular edema, and serous retinal detachment. We present a case of acute bilateral anterior uveitis, prolonged hypotony, and cataracts following the use of dual therapy ipilimumab and nivolumab. Physicians should be aware of these immune-mediated ocular adverse events and should have a management plan to deal with these side effects that range from mild to vision threatening.




\section{Case Reports in Ophthalmology}

\section{Introduction}

Immune checkpoint inhibitors (ICIs) are a relatively new class of immunomodulating agents transforming anti-cancer therapy. These medications block inhibitory receptors of the immune system including (a) cytotoxic T-lymphocyte-associated antigen-4 (CTLA-4) and (b) programmed cell death protein-1 (PD-1) and its ligands (PDL-1 and PDL-2), leading to T-cell activation and enhancement of the immune response against tumor cells. Since 2011, the Food and Drug Administration (FDA) has approved a number of ICI therapies, including immunoglobulin G1 (IgG1) anti-CTLA-4 human monoclonal antibody ipilimumab (Yervoy, Bristol-Myers Squibb, New York, NY, USA) and the IgG4 anti-PD-1 monoclonal antibody nivolumab (Opdivo, Bristol-Myers Squibb) for various malignancies, such as advanced melanoma, nonsmall-cell lung cancer, squamous cell carcinoma of the head and neck, renal cell carcinoma, urothelial carcinoma, and Hodgkin lymphoma. Clinical trials have yielded high response rates but also reported frequent immune-related adverse effects (IRAEs) [1].

Patients receiving ICIs develop IRAEs at a 10-15\% higher rate compared with traditional chemotherapeutic drugs [1]. The adverse effects most commonly affect the dermatologic, gastrointestinal, hepatic, and endocrine systems [1]. However, while ophthalmic side effects secondary to ICI use are rare, occurring in approximately $1 \%$ of patients, these effects can be significant and vision-threatening, including dry eyes, episcleritis, peripheral ulcerative keratitis, uveitis, inflammatory orbitopathy, myasthenia gravis, macular edema, serous retinal detachment, choroidal neovascularization, and Vogt-Koyanagi-Harada (VKH) reaction [1-3].

In 2018, the FDA granted approval of ipilimumab and nivolumab combination therapy for the treatment of intermediate or poor risk, previously untreated advanced renal cell carcinoma. Little is known regarding the occurrence and management of ocular IRAEs associated with the newer combination therapies.

We present a case of acute bilateral anterior uveitis, prolonged hypotony, and nuclear cataracts associated with dual immunotherapy ipilimumab and nivolumab and discuss the ways to evaluate, manage, and treat these side effects.

\section{Case Report}

In mid-September 2019, a 71-year-old man with a history of stage IV clear cell renal cell carcinoma, diabetes, and hypertension was referred to us by his local oncologist and optometrist for new-onset dry, red, and sore eyes, as well as blurred vision. One week prior, the patient was started on ipilimumab plus nivolumab therapy. Previously in June 2019 during his hospitalization for acute renal failure, persistent hypercalcemia, metabolic encephalopathy, and a left renal mass, he was diagnosed with renal cancer. A partial nephrectomy revealed grade III clear cell carcinoma with positive margins. Subsequent staging imaging showed an isolated, non-calcified 5-mm pulmonary nodule felt to be metastatic disease-causing hypercalcemia of malignancy. In early September 2019, he received his first cycle of ipilimumab plus nivolumab. As part of his oncologic management, the patient was also prescribed prednisone $10 \mathrm{mg}$ daily for 8 weeks during the course of his chemotherapy. Prior to this, the patient had not received corticosteroids.

On presentation, his best corrected visual acuity was 20/70 bilaterally, and intraocular pressure (IOP) was $8 \mathrm{~mm} \mathrm{Hg}$ in the right eye and $5 \mathrm{~mm} \mathrm{Hg}$ in the left eye. Slit-lamp exam was remarkable for bilateral $1+$ conjunctival injection, 1+ keratic precipitates, $1+$ cell, posterior synechiae, mid-dilated pupils, and 2+ nuclear scleroses (Fig. 1a, b). There was no evidence of 


\section{Case Reports in Ophthalmology}

vitreous involvement bilaterally. Fundus exam revealed the right macula had cotton-wool spots and hard exudates, while both eyes demonstrated arteriolar narrowing consistent with grade III hypertensive retinopathy (Modified Scheie Classification) with no macular edema (Fig. 1c, d). Disk size appeared small (disks at risk) with cup to disk ratios of 0.1 in the right eye and 0.25 in the left eye, including bilateral peripapillary atrophy. Optical coherence tomography (OCT) horizontal B-scan imaging showed optic nerve head crowding (Fig. 1e). Extraocular movements and visual fields were normal. Prior optometric records were normal.

OCT angiography (OCTA; Optovue Inc., Fremont, CA, USA) revealed normal nerve fiber layer thickness and small disks (Fig. 2a). Vessel density in the macula and around the optic nerve were decreased bilaterally (Fig. 2b, c). Disk diameters were $1.56 \mathrm{~mm}$ in the right and $1.73 \mathrm{~mm}$ in the left. A diagnosis of bilateral granulomatous anterior uveitis, hypotony, and nuclear cataracts was made. The patient was asked to continue with local anti-inflammatory treatment started by his optometrist (Pred Forte ${ }^{\circledR}$ [prednisolone acetate] ophthalmic suspension $1 \%$ ) every $3 \mathrm{~h}$ bilaterally, along with atropine sulfate $1 \%$ twice daily. At 5 days follow-up, the patient's visual acuity improved to 20/50 in the right eye and 20/40 in the left eye with improved eye redness. After 2 weeks, atropine was discontinued and Pred Forte ${ }^{\circledR}$ drops were decreased to four times daily. After 5 weeks, the steroid eye drops were decreased to twice daily bilaterally. At the patient's most recent follow-up appointment, 10 weeks after initial presentation, the Pred Forte ${ }^{\circledR}$ drops were decreased to once daily in both eyes. His eye redness had resolved. Visual acuity gradually rose to 20/40 and 20/25 in the right and left eyes, respectively. IOP increased to $9 \mathrm{~mm} \mathrm{Hg}$ bilaterally. There were no signs of continual anterior chamber inflammation, and the status of the cataracts remained stable in both eyes. The patient completed four cycles of ipilimumab plus nivolumab before he was transitioned to maintenance nivolumab monotherapy in January 2020 (15 weeks after his initial ophthalmic presentation).

\section{Discussion}

The relatively new class of ICIs has revolutionized the treatment of several cancers. However, ICIs have also been associated with a number of adverse events, most commonly dermatitis, colitis, hepatitis, and hypophysitis [1, 4]. Ophthalmologic adverse events are rare, commonly cited as less than $1 \%$ of all patients, but well-recognized [1-3]. Tarhini [5] found that ocular IRAEs occurred in $1.3 \%$ of patients receiving ipilimumab, whereas Zimmer et al. [6] reported an incidence of $1.6 \%$ of patients receiving anti-PD-1 treatment (nivolumab or pembrolizumab). While several ocular IRAEs have been reported, the most common are uveitis $(0.3-6 \%$, anterior more common than posterior or panuveitis) and dry eyes (1-24\%) [1-3].

In regards to the combination of ipilimumab and nivolumab, the ocular IRAEs described include dry eyes, corneal punctate epithelial erosions, subconjunctival hemorrhage, spontaneous corneal perforation, uveitis, hypotony maculopathy, cystoid macular edema, serous retinal detachment, choroiditis, VKH syndrome, optic neuritis, melanoma-associated retinopathy, and ocular myasthenia gravis [7, 8]. In 2 studies across 8 institutions, nearly all cases of ipilimumab-nivolumab associated uveitis were bilateral eye involvement and mild (trace cell) to moderate (1-2+ cell) in severity $[7,8]$.

Ocular IRAEs typically occur early in the immunotherapy course; however, delayed reactions have also been observed, with a median occurrence of 2 months after checkpoint inhibitor initiation [1, 5, 9]. Given our patient's temporal relationship between starting 


\section{Case Reports in Ophthalmology}

immunotherapy and presenting with bilateral anterior uveitis, we diagnosed him with a druginduced complication.

Our patient's prolonged hypotony may have been from uveitis, but other causes of hypotony include trauma, medications, and ocular ischemia [10]. Nine months before this presentation, our patient's IOPs were 20- and 21-mm Hg in the right and left eyes, respectively. OCTA revealed low vessel density in both eyes most likely from his vasculopathic risk factors of hypertension and diabetes (Fig. 2a-c). We also postulate that the combination immunotherapy may have played a role in reducing blood supply to the ciliary body, resulting in ciliary body shutdown.

The patient's 2+ nuclear cataracts may have been accelerated by uveitis and poor general health. While topical and systemic corticosteroids are known to cause cataracts, they are typically of the posterior subcapsular type and are usually associated with higher dose and longer duration of systemic corticosteroids (>10 mg daily for over a year) [11]. Our patient took a relatively low dose of prednisone $10 \mathrm{mg}$ daily for a short period, and presented with nuclear sclerotic cataracts, less consistent with the use of corticosteroids. Additionally, his cataracts were noted on our exam prior to his taking any corticosteroids. With treatment and resolution of his uveitis, the patient's vision improved but his cataracts persisted.

The treatment of ophthalmic IRAEs depends on the severity and the type of symptoms. Topical corticosteroids are typically used in mild to moderate presentations (anterior uveitis and episcleritis), whereas systemic treatment is indicated for more severe ocular inflammation (intermediate or posterior uveitis, VKH syndrome, and inflammatory orbitopathy) $[1,3$, $9,12]$. Consultation with a rheumatologist and oncologist is critical. Current oncologic guidelines recommend taking an immunotherapy holiday for any grade III adverse event (anterior uveitis with $3+$ or greater cells; intermediate, posterior, or panuveitis) until resolved and permanent discontinuation for grade IV events (visual acuity of 20/200 or worse in the affected eye) $[13,14]$.

Complete resolution of our patient's ocular inflammation and symptoms was achieved with topical corticosteroids and cycloplegia, and he did not have to discontinue immunotherapy. More aggressive forms of systemic or invasive measures and rheumatologic consultation were not sought as the patient responded well to initial therapy.

\section{Conclusion}

We report a triad of ocular complications consisting of bilateral uveitis, prolonged hypotony, and cataracts following the use of ipilimumab and nivolumab. Oncologists should be aware of these ocular IRAEs and refer these patients appropriately to an ophthalmologist. Coordination among oncologists, ophthalmologists, and rheumatologists is essential to preserve patients' general health and eyesight. Early ophthalmologic evaluation and management can result in good visual outcomes.

\section{Statement of Ethics}

This case report was conducted according to good clinical practice. Written informed consent was obtained from the patient for publication of this article and any accompanying images. The authors state that they have full control over all primary data and have no ethical conflicts to disclose. 


\section{Case Reports in Ophthalmology}

\section{Conflict of Interest Statement}

The authors have no conflicts of interest to declare.

\section{Funding Sources}

Supported in part by an unrestricted grant from the Research to Prevent Blindness, New York, NY, and Visual Sciences Core Grant EY020799.

\section{Author Contributions}

All authors have been involved in drafting the manuscript or revising it critically and have given final approval of this version to be published and agreed to be accountable for all aspects of the work.

\section{References}

1 Antoun J, Titah C, Cochereau I. Ocular and orbital side-effects of checkpoint inhibitors: a review article. Curr Opin Oncol. 2016 Jul;28(4):288-94.

2 Abdel-Rahman O, Oweira H, Petrausch U, Helbling D, Schmidt J, Mannhart M, et al. Immune-related ocular toxicities in solid tumor patients treated with immune checkpoint inhibitors: a systematic review. Expert Rev Anticancer Ther. 2017 Apr;17(4):387-94.

3 Dalvin LA, Shields CL, Orloff M, Sato T, Shields JA. CHECKPOINT INHIBITOR IMMUNE THERAPY: Systemic Indications and Ophthalmic Side Effects. Retina. 2018 Jun;38(6):1063-78.

4 Fang T, Maberley DA, Etminan M. Ocular adverse events with immune checkpoint inhibitors. J Curr Ophthalmol. 2019 Jun;31(3):319-22.

5 Tarhini A. Immune-mediated adverse events associated with ipilimumab ctla-4 blockade therapy: the underlying mechanisms and clinical management. Scientifica (Cairo). 2013;2013:857519.

6 Zimmer L, Goldinger SM, Hofmann L, Loquai C, Ugurel S, Thomas I, et al. Neurological, respiratory, musculoskeletal, cardiac and ocular side-effects of anti-PD-1 therapy. Eur J Cancer. 2016 Jun;60:210-25.

7 Kim JM, Materin MA, Sznol M, Kluger HM, Weiss S, Chow J, et al. Ophthalmic Immune-Related Adverse Events of Immunotherapy: A Single-Site Case Series. Ophthalmology. 2019 Jul;126(7):1058-62.

8 Sun MM, Levinson RD, Filipowicz A, Anesi S, Kaplan HJ, Wang W, et al. Uveitis in Patients Treated with CTLA4 and PD-1 Checkpoint Blockade Inhibition. Ocul Immunol Inflamm. 2020;28(2):217-27.

9 Bitton K, Michot JM, Barreau E, Lambotte O, Haigh O, Marabelle A, et al. Prevalence and Clinical Patterns of Ocular Complications Associated With Anti-PD-1/PD-L1 Anticancer Immunotherapy. Am J Ophthalmol. 2019 Jun;202:109-17.

10 Wang Q, Thau A, Levin AV, Lee D. Ocular hypotony: A comprehensive review. Surv Ophthalmol. 2019 Sep Oct;64(5):619-38.

11 Crews SJ. Posterior subcapsular lens opacities in patients on longterm corticosteroid therapy. BMJ. 1963 Jun;1(5346):1644-7.

12 Rémond AL, Barreau E, Le Hoang P, Bodaghi B. [Bilateral uveitis associated with nivolumab therapy]. J Fr Ophtalmol. 2018 Jun;41(6):536-9.

13 Brahmer JR, Lacchetti C, Schneider BJ, Atkins MB, Brassil KJ, Caterino JM, et al.; National Comprehensive Cancer Network. Management of Immune-Related Adverse Events in Patients Treated With Immune Checkpoint Inhibitor Therapy: American Society of Clinical Oncology Clinical Practice Guideline. J Clin Oncol. 2018 Jun;36(17):1714-68.

14 Puzanov I, Diab A, Abdallah K, Bingham CO 3rd, Brogdon C, Dadu R, et al.; Society for Immunotherapy of Cancer Toxicity Management Working Group. Managing toxicities associated with immune checkpoint inhibitors: consensus recommendations from the Society for Immunotherapy of Cancer (SITC) Toxicity Management Working Group. J Immunother Cancer. 2017 Nov;5(1):95. 


\section{Ophthalmology}
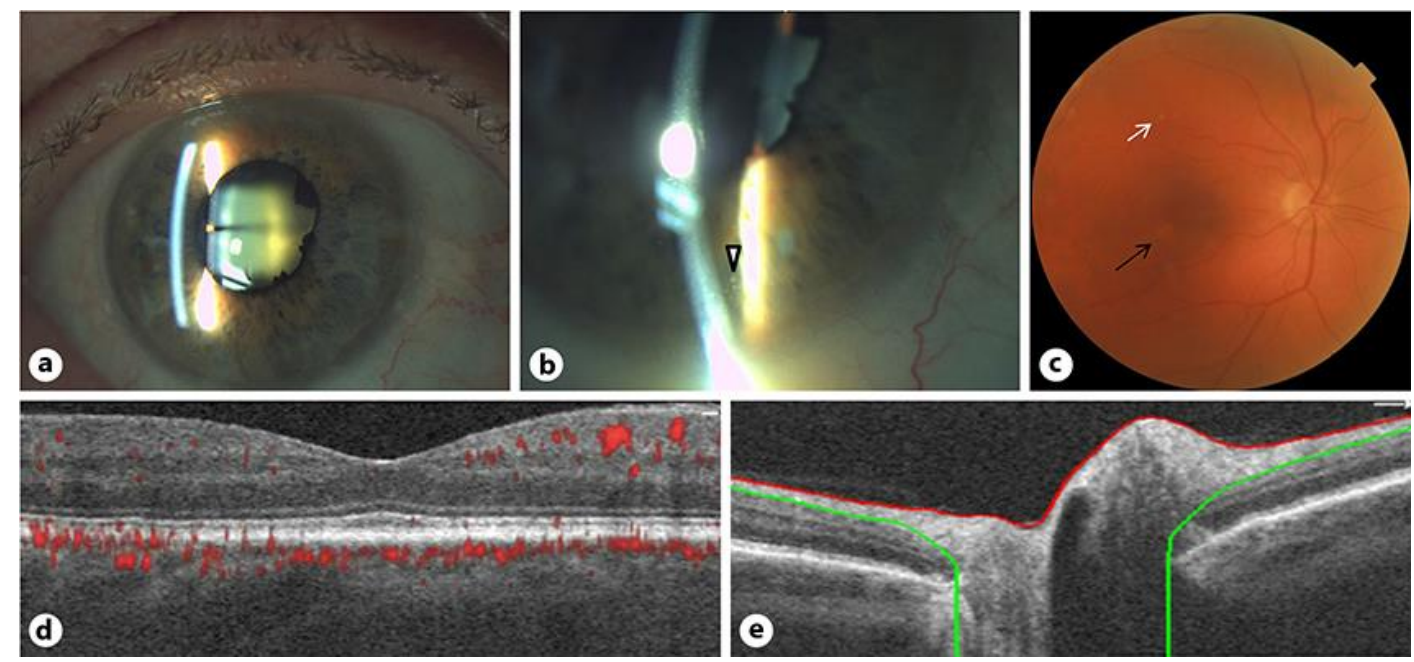

Fig. 1. Slit lamp, fundus, and OCT horizontal B-scan images (right eye). a, b Slit lamp photographs showing posterior synechiae and nuclear cataract (a) and keratic precipitates (b) (white triangle). c Fundus photograph showing cup-to-disk ratio of 0.1 , small diameter $(1.56 \mathrm{~mm})$, peripapillary atrophy, narrow arterioles, cotton wool spots (black arrow), and hard exudates (white arrow). d, e OCT horizontal B-scan imaging with normal retina and choroid and no evidence of retinal edema (d) and optic nerve head crowding (e).
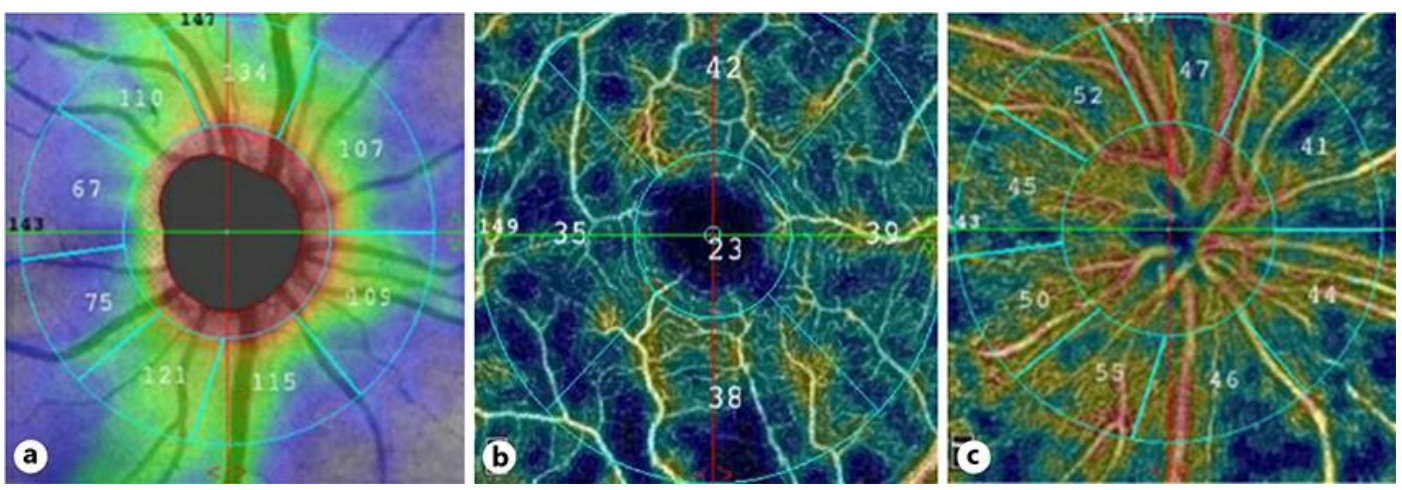

Fig. 2. OCTA scans showing normal nerve fiber layer thickness (a), macula with reduced vessel density, average density $38.5 \%$ (normal $\geq 50 \%$ ) (b), and optic nerve with mildly reduced vessel density, average density $47.5 \%$ (normal $\geq 50 \%$ ) (c). 